\title{
Uma Revisão Sobre a Formação Docente para o Ensino-Aprendizagem do Raciocínio Computacional no Brasil
}

\author{
Ana Carolina Sokolonski ${ }^{1}$, Alirio Santos de Sá $^{1}$, Raimundo José de Araújo Macêdo ${ }^{1}$ \\ ${ }^{1}$ Laboratório de Sistemas Distribuídos (LaSiD) \\ Programa de Pós-Graduação em Mecatrônica (PPGM) \\ Universidade Federal da Bahia (UFBA) \\ Av. Adhemar de Barros S/N - Salvador, BA - Brasil \\ carolsoko@ifba.edu.br, aliriosa@ufba.br, macedo@ufba.br
}

\begin{abstract}
Several teaching-learning approaches for Computational Thinking (CT) have been proposed and investigated in the last years. Recently, with the Brazilian National Common Curricular Base (BNCC) for primary and secondary education, the teaching-learning of CT became a significant challenge due to the need for adequate teacher training to meet the imminent demand. This article presents a systematic literature review about existing and published approaches for the training of teachers for CT in Brazil. The selected publications were classified and analyzed considering publication year, type of study, teaching-learning approaches, target audience, taught CT skills, and online education. Lastly, based on the analyses, we highlight advantages and disadvantages, challenges, and future works.
\end{abstract}

Resumo. Nos últimos anos, diversas abordagens de ensino-aprendizagem de Raciocínio ou Pensamento Computacional têm sido propostas e investigadas. Recentemente, com a publicação da Base Nacional Comum Curricular (BNCC) para o Ensino Básico e suas prescritas competências, o ensino-aprendizagem do Raciocínio Computacional $(R C)$ tornou-se um grande desafio a ser superado, tendo em vista a necessidade de formação docente adequada para atender a enorme demanda iminente. Neste artigo, através de uma revisão sistemática de literatura dos trabalhos publicados existentes na literatura, foi traçado um cenário da formação de docentes para o ensino-aprendizagem do Raciocínio Computacional no Brasil. Para tanto, as publicações selecionadas foram classificadas e analisadas por ano de publicação, tipo de estudo, abordagem de ensino-aprendizagem utilizada, público-alvo investigado, habilidades do RC desenvolvidas e uso de ensino online. Por fim, baseado nas análises, destacamse as vantagens e desvantagens, desafios e trabalhos futuros.

\section{Introdução e conceitos iniciais}

A revolução digital nas últimas décadas promoveu uma mudança profunda na forma como as pessoas vivem e se relacionam. Nos últimos anos, a computação passou a integrar os processos de desenvolvimento e produção em uma grande diversidade de profissões, exigindo de seus profissionais conhecimento e domínio de conceitos básicos, anteriormente somente atribuídos aos cientistas ou profissionais da computação. Além disso, a computação tem uma forma própria de lidar com processos complexos, viabilizando não apenas 
uma estruturação do problema que facilita a compreensão, mas também um planejamento e organização de estratégias eficientes. Nesse sentido, o Pensamento ou Raciocínio Computacional $^{1}$ passou a ter cada vez mais relevância na formação das pessoas, independente de sua área de formação.

As novas formas, mídias e significados construídos nas últimas três décadas a partir do advento da era digital e do surgimento da Internet, em particular, nos trouxeram a um cenário de acesso, gestão e produção de informações sem precedentes, revolucionando a natureza dos problemas que enfrentamos e requerendo articulação e integração de saberes e práticas de campos diversos. Como alerta o filósofo Michel Serres, em sua obra intitulada Polegarzinha (2013), precisamos estar atentos tanto às habilidades que estão deixando de ser necessárias quanto às novas habilidades que estão sendo desenvolvidas pelos nossos jovens em decorrência da revolução que nos proporciona o fácil acesso à informação [Macêdo et al. 2020, pag 420].

O Raciocínio Computacional é definido por alguns pesquisadores como uma maneira de organizar o raciocínio, podendo explorar habilidades de matemática, raciocínio lógico, resolução e abstração de problemas, entre outras [Wing 2006]. Jeannette Wing defende que qualquer pessoa pode e deve desenvolver e se apropriar desta forma de pensar, pois os benefícios de pensar como um cientista da computação vão além de saber operar um computador e não estão restritos aos problemas computacionais, lógica e matemática, pois abrangem todas as áreas do conhecimento e ações do dia-a-dia, relativas ao planejamento e soluções eficazes para resolução de problemas e desafios.

O Raciocínio Computacional desenvolve a capacidade de compreender, definir, modelar, comparar, solucionar, automatizar e analisar problemas e possíveis soluções de forma metódica e sistemática por meio da construção de algoritmos [SBC 2020]. As 4 principais habilidades do Raciocínio Computacional são: abstração, decomposição, reconhecimento de padrões e raciocínio algorítmico. O processo de ensino-aprendizagem do Raciocínio Computacional visa desenvolver nos alunos a capacidade de organizar o raciocínio com o objetivo de solucionar problemas complexos de forma rápida e simples.

A Base Nacional Comum Curricular (BNCC), que fundamenta toda a educação básica brasileira, em sua última revisão, publicada em 2018, incorporou competências que tangenciam o Raciocínio Computacional. Destaca-se o seguinte trecho da BNCC:

São definidas competências e habilidades, nas diferentes áreas, que permitem aos estudantes:[...]utilizar, propor e/ou implementar soluções (processos e produtos) envolvendo diferentes tecnologias, para identificar, analisar, modelar e solucionar problemas complexos em diversas áreas da vida cotidiana, explorando de forma efetiva o raciocínio lógico, o pensamento computacional, o espírito de investigação e a criatividade. [Brasil 2020, pag 475]

Nota-se que, para atender às exigências da BNCC para o ensino básico, é necessário que o aluno aprenda diversos conteúdos, entre eles o Raciocínio Computacional, demandando

\footnotetext{
${ }^{1}$ Do inglês Computational Thinking
} 
que os docentes sejam preparados para fomentar o desenvolvimento de tais conteúdos na educação básica.

Este trabalho tem o objetivo de avaliar, através de uma revisão sistemática de literatura, como a formação docente para o ensino-aprendizagem do Raciocínio Computacional vem sendo tratada no Brasil. A presente revisão considerou publicações de trabalhos realizados com a formação docente inicial, que são os trabalhos que contemplam os cursos de licenciaturas, e com a formação docente continuada, que são os trabalhos que envolvem docentes formados.

Na Seção 2, será apresentada a Revisão Sistemática da Literatura realizada, destacando as informações mais relevantes. Na Seção 3, serão discutidos os resultados. Por fim, a conclusão do estudo e trabalhos futuros serão apresentados na Seção 4.

\section{Revisão Sistemática da Literatura}

Entre as metodologias existentes para a realização de revisões sistemáticas da literatura, a presente revisão se baseou na metodologia de Kitchenham [Kitchenham 2004]. A seguinte pergunta-chave foi norteadora desta pesquisa: "O que tem sido proposto para a formação de professores para o ensino de Raciocínio Computacional no Brasil?".

A partir desta pergunta, definiu-se o padrão de busca da seguinte forma: ((formação de professores OR formação docente) AND (Raciocínio Computacional OR Pensamento Computacional)) OR ((teacher training OR teacher education) AND Computational Thinking AND Brazil). A partir deste padrão de busca, foram realizadas pesquisas nas bases de dados, considerando trabalhos publicados até o dia 05 de março de 2021, e os resultados podem ser observados na Tabela 1. As bases de dados escolhidas foram o Periódicos da CAPES, Google Scholar e ResearchGate, pois estas bases retornam publicações nacionais e internacionais de variadas fontes, obtendo acesso a publicações em português e inglês dos maiores eventos, revistas e universidades do mundo.

Tabela 1. Resultado da Busca nas Bases de Dados

\begin{tabular}{||c|c|c||}
\hline \hline Base de Dados & URL & Qtde de Estudos Encontrados \\
\hline \hline Periódicos da CAPES & periodicos.capes.gov.br & 94 \\
\hline Google Scholar & scholar.google.com & 2684 \\
\hline Research Gate & www.researchgate.net & 1370 \\
\hline \hline Total de estudos & - & 4148 \\
\hline \hline
\end{tabular}

A triagem dos estudos eliminou trabalhos duplicados, sem fonte de publicação, que não possuíam sua versão completa disponível, que não foram publicados em português ou inglês, ou que não possuíam as palavras-chave em seu título ou resumo. Após ler o título e o resumo dos trabalhos, sempre focando nos trabalhos que envolvessem a formação docente para o ensino-aprendizagem do Raciocínio Computacional, foram selecionados 39 trabalhos, que foram lidos na íntegra e classificados. A Tabela 2 apresenta, em ordem cronológica, os 39 estudos selecionados.

\subsection{Extração de Dados e Meta-Análise}

Os trabalhos foram classificados quanto ao ano de publicação, tipo de estudo realizado, estratégias de ensino-aprendizagem utilizadas, público investigado, habilidades do Racio- 
Tabela 2. Listagem de Estudos Elegíveis

\begin{tabular}{|c|c|c|}
\hline Ano Publicação & Referências & Total por ano \\
\hline 2014 & [Ramos and Espadeiro 2014] & 1 \\
\hline 2015 & [Farias et al. 2015] & 1 \\
\hline 2016 & $\begin{array}{c}\text { [Souza et al. 2016, Kampff et al. 2016] } \\
\text { [Valente 2016, Barcelos et al. 2016] }\end{array}$ & 4 \\
\hline 2017 & $\begin{array}{c}\text { [Paz 2017, Costa et al. 2017] } \\
\text { [von Wangenheim et al. 2017, Eloy et al. 2017] } \\
\text { [França and Tedesco 2017, Silva et al. 2017] }\end{array}$ & 6 \\
\hline 2018 & $\begin{array}{c}\text { [Barros et al. 2018b, Barros et al. 2018a] } \\
\text { [Pasqual Júnior 2018, Mandaji et al. 2018] } \\
\text { [Vasconcelos and Neto 2018] }\end{array}$ & 5 \\
\hline 2019 & $\begin{array}{c}\text { [Matos et al. 2019, Marques et al. 2019, Barbosa 2019] } \\
\text { [Souza 2019, Goulart et al. 2019, Silva et al. 2019] } \\
\text { [Júnior and de Oliveira 2019, Lira et al. 2019] } \\
\text { [Oliveira and Bittencourt 2019, Oliveira et al. 2019] } \\
\text { [Oliveira and Pereira 2019, Zilio and Nóbile 2019] }\end{array}$ & 12 \\
\hline 2020 & $\begin{array}{c}\text { [Pimentel et al. 2020, Martins and Giraffa 2020] } \\
\text { [Barbosa and Maltempi 2020, Agnol et al. 2020] } \\
\text { [Macêdo et al. 2020, Côrrea et al. 2020] } \\
\text { [Reichert et al. 2020, Gomes et al. 2020] } \\
\text { [Echenique and Fontes 2020] }\end{array}$ & 9 \\
\hline 2021 & {$[$ Menolli et al. 2021] } & 1 \\
\hline Total de estudos & - & 39 \\
\hline
\end{tabular}

cínio Computacional desenvolvidas, e se as propostas envolveram formação online. Estas dimensões foram escolhidas por nos proporcionar uma visão clara e objetiva da maneira como a formação docente vem sendo proposta ao longo dos últimos anos no Brasil.

A revisão sistemática considerou publicações entre 01 de janeiro 2014 e 05 de março de 2021. A maioria dos trabalhos foram publicados nos anos de 2019 e 2020, como pode ser visto na Tabela 2, o que mostra o quanto o tema é atual. Dentre os 39 estudos selecionados, 32 foram estudos que envolveram aplicações práticas e 7 foram estudos que não envolveram aplicações práticas, conforme Tabela 3. Nesta relação, estudos que envolveram aplicações práticas são desde estudos de casos e relatos de experiências, até propostas de abordagens, validadas através de oficinas, cursos ou experimentações.

Os estudos utilizaram diversas estratégias para a formação docente no ensinoaprendizagem do Raciocínio Computacional. Pode-se observar que a estratégia mais utilizada foi Ensino de Lógica de Programação, 61,54\%, seguida da Computação Desplugada, 25,64\%, e Gamificação, 23,08\%, conforme Tabela 4. Como muitos estudos utilizaram mais de uma estratégia, o somatório ultrapassa $100 \%$. Além disso, alguns estudos não informaram a estratégia utilizada.

A modificação do currículo proposta pela BNCC sugere que o Raciocínio Computacional faça parte do ensino básico brasileiro, portanto, uma mudança na formação do- 
Tabela 3. Tipos de Estudos Analisados

\begin{tabular}{||c|c|c||}
\hline \hline Tipos de Estudos & Quantidade de Estudos & Percentual do Total \\
\hline \hline Estudos COM Aplicações Práticas & 32 & $82,05 \%$ \\
\hline Estudos SEM Aplicações Práticas & 7 & $17,95 \%$ \\
\hline \hline Total de estudos & 39 & $100 \%$ \\
\hline \hline
\end{tabular}

Tabela 4. Estratégias Adotadas no Ensino-Aprendizagem do Raciocínio Computacional

\begin{tabular}{||c|c|c||}
\hline \hline Tipos de Estratégia & Quantidade de Estudos & Percentual do Total \\
\hline \hline Robótica Educacional & 7 & $17,95 \%$ \\
\hline Ensino de Lógica de Programação & 24 & $61,54 \%$ \\
\hline Gamificação & 9 & $23,08 \%$ \\
\hline Computação Desplugada & 10 & $25,64 \%$ \\
\hline \hline Total de estudos & 39 & $100 \%$ \\
\hline
\end{tabular}

cente, inicial e continuada, faz-se necessária. Conforme apontam vários autores, docentes em formação continuada, ou seja, que buscam cursos de aprimoramento após sua formação básica inicial, frequentemente mostram-se resistentes ao aprendizado do Raciocínio Computacional, seja por não entenderem a sua importância, seja por possuírem dificuldades com o uso das tecnologias [Barros et al. 2018a, Júnior and de Oliveira 2019].

Tabela 5. Formação Inicial X Formação Continuada

\begin{tabular}{||c|c|c||}
\hline \hline Tipos de Público & Quantidade de Estudos & Percentual do Total \\
\hline \hline Formação Inicial & 18 & $46,15 \%$ \\
\hline Formação Continuada & 18 & $46,15 \%$ \\
\hline Ambas & 3 & $7,70 \%$ \\
\hline \hline Total de estudos & 39 & $100 \%$ \\
\hline \hline
\end{tabular}

Nos trabalhos encontrados, 46,15\% investigaram apenas a formação inicial, $46,15 \%$ investigaram apenas a formação continuada e 7,70\% investigaram ambas formações, como pode ser visto na Tabela 5. O que mostra que os esforços para a modificação necessária na formação continuada estão acontecendo e precisam continuar avançando.

Apesar de 26 estudos, 66,67\% do total, definirem as habilidades do Raciocínio Computacional em sua fundamentação teórica, apenas 48,72\% deles avaliaram as habilidades desenvolvidas nos experimentos. Das habilidades avaliadas, as quatro habilidades básicas são as mais investigadas, como visto na Tabela 6 . Apenas 28,21\% dos trabalhos investigaram outras habilidades, além das 4 principais.

Dos 39 estudos selecionados, apenas 6 contemplaram propostas de formação online de docentes, o que representa $15,38 \%$ do total, ver Tabela 7 . A formação online mostrou-se, nos últimos anos, necessária para o ensino em todo o mundo. Os professores tiveram que se adaptar ao ensino online, que traz a educação remota por meios digitais para o protagonismo do dia-a-dia docente. 
Tabela 6. Habilidades do Raciocínio Computacional Analisadas

\begin{tabular}{||c|c|c||}
\hline \hline Habilidade & Quantidade de Estudos & Percentual do Total \\
\hline \hline Abstração & 19 & $48,72 \%$ \\
\hline Decomposição & 18 & $46,15 \%$ \\
\hline Raciocínio Algorítmico & 18 & $46,15 \%$ \\
\hline Reconhecimento de Padrões & 19 & $48,72 \%$ \\
\hline Outras habilidades & 11 & $28,21 \%$ \\
\hline \hline Total de estudos & 39 & $100 \%$ \\
\hline \hline
\end{tabular}

Tabela 7. Formato de Apresentação das Aulas

\begin{tabular}{||c|c|c||}
\hline \hline Tipos de Formação Proposta & Quantidade de Estudos & Percentual do Total \\
\hline \hline Formação Online & 6 & $15,38 \%$ \\
\hline Formação Presencial & 33 & $84,62 \%$ \\
\hline \hline Total de estudos & 39 & $100 \%$ \\
\hline \hline
\end{tabular}

\section{Discussão dos Resultados}

A partir das análises realizadas, observa-se que diversas estratégias de ensino vêm sendo utilizadas na formação docente para o ensino-aprendizagem do Raciocínio Computacional. A estratégia mais utilizada é o Ensino de Lógica de Programação, seguida pela Computação Desplugada, Gamificação e Robótica Educacional, nesta ordem.

A maior parte dos trabalhos que adotaram o Ensino de Lógica de Programação utilizaram Softwares como o Scratch e o App Inventor para estimular a participação dos docentes, ensinando lógica através de blocos, alguns incorporando a Gamificação. Esta abordagem para o ensino-aprendizagem do Raciocínio Computacional desenvolve as quatro habilidades básicas (i.e., abstração, decomposição, generalização e raciocínio algorítmico), porém associa o aprendizado do Raciocínio Computacional à Programação de Computadores, impossibilitando sua aplicação na primeira infância, por exemplo, e trazendo um viés técnico muito forte.

A Robótica Educacional é um exemplo de abordagem que promove o aprendizado lúdico e multidisciplinar, possibilitando o uso em diversos níveis de ensino, podendo estimular habilidades como trabalho em grupo, auto-análise e auto-crítica [Sokolonski et al. 2020]. Esta abordagem foi pouco explorada, sendo utilizada em apenas 7 trabalhos, sem proposta concreta para incorporação ao currículo das licenciaturas.

Uma proposta feita para a formação docente pode ter reflexos no ensino básico, pois os docentes tendem a reproduzir as técnicas aprendidas. Portanto, deve-se explorar técnicas que sejam compatíveis com todos os níveis de ensino, diversificando-as. Fatores como custo e aplicabilidade devem ser avaliados. Sabe-se que a Robótica Educacional requer um investimento elevado, assim, técnicas como a Computação Desplugada são mais viáveis em escolas públicas, por exemplo. Porém, caso haja recursos disponíveis, técnicas como Gamificação e Robótica Educacional são mais atraentes aos jovens.

Outro aspecto que deve ser investigado é se as propostas de ensino-aprendizagem realizadas estão sendo formalmente adotadas nos cursos de licenciaturas e formação con- 
tinuada. Para isto, um trabalho futuro interessante seria investigar os planos pedagógicos das licenciaturas brasileiras com o objetivo de obter tais informações. A situação do ensino-aprendizagem do Raciocínio Computacional nas licenciaturas do Brasil é uma informação que não é fácil de ser conseguida, porém, precisa ser investigada.

Por fim, faz-se necessário informar que toda revisão sistemática da literatura está sujeita a vulnerabilidades, que ameaçam a validade de seus resultados, pois esta é passível de erros humanos, conferindo um grau de imprecisão na mesma.

\section{Conclusão}

Hoje, tanto a formação docente inicial quanto a continuada precisam ser investigadas. Não basta atualizar o conteúdo programático dos cursos de licenciatura, necessita-se, também, capacitar os professores que estão atuando no ensino básico. A resistência em mudar é muitas vezes uma barreira do público da formação continuada, porém, no cenário proposto pela BNCC, onde o ensino básico deverá sofrer alterações significativas nos próximos anos, essa mudança é necessária. Assim, deve-se investigar melhor a formação docente continuada, visando prover ferramentas para incentivar o aprendizado.

A avaliação das habilidades do Raciocínio Computacional desenvolvidas nos experimentos é uma importante dimensão, pois garante que o Raciocínio Computacional foi compreendido adequadamente pelos docentes. Menos de 50\% dos estudos fizeram avaliações sobre quais habilidades foram desenvolvidas em seus experimentos. Dos estudos avaliados, apenas $28,21 \%$ avaliaram mais que as 4 principais. A avaliação das habilidades do Raciocínio Computacional podem oportunizar propostas de melhorias do processo ensino-aprendizagem e desenvolvimento de material didático direcionado às necessidades do público docente, seja em formação inicial ou continuada.

Por fim, a formação online docente precisa ser investigada. Apenas 6 estudos trabalharam com essa modalidade de ensino, que se mostrou tão necessária e emergente no último ano, devido à pandemia. Assim, estudos que envolvam formação docente online para o ensino-aprendizagem do Raciocínio Computacional são necessários.

\section{Referências}

Agnol, A. D., Gusberti, C., and Bertagnolli, S. C. (2020). O Ensino de Pensamento Computacional Através de um Jogo de Tabuleiro em Ambiente Desplugado: Relato de Experiência de Formação Docente. Revista Novas Tecnologias na Educação, 18(1).

Barbosa, L. (2019). A inserção do Pensamento Computacional na Base Nacional Comum Curricular: reflexões acerca das implicações para a formação inicial dos professores de matemática. Anais do XXV Workshop de Informática na Escola, 25:889-898.

Barbosa, L. L. d. S. and Maltempi, M. (2020). Matemática, Pensamento Computacional e BNCC: Desafios e Potencialidades dos Projetos de Ensino e das Tecnologias na Formação Inicial de Professores. Revista Brasileira de Ensino de Ciências e Matemática, 3(3).

Barcelos, T., Bortoletto, R., and Andrioli, M. (2016). Formação Online para o Desenvolvimento do Pensamento Computacional em Professores de Matemática. Anais dos Workshops do Congresso Brasileiro de Informática na Educação, 5:1228. 
Barros, T., Reategui, E., Radaelli, R., and Teixeira, A. (2018a). Análise de Discurso e de Conteúdo de uma Formação em Pensamento Computacional para Professores. Simpósio Brasileiro de Informática na Educação - SBIE, 29:1733.

Barros, T. T. T., Reategui, E. B., Meira, R. R., and Teixeira, A. C. (2018b). Avaliando a Formação de Professores no Contexto do Pensamento Computacional. Revista Novas Tecnologias na Educação, 29.

Brasil (2020). Base Nacional Comum Curricular (BNCC). Ministério da Educação, Brasília-Brasil. http://basenacionalcomum.mec.gov.br/.

Côrrea, E. B. et al. (2020). O Desenvolvimento do Pensamento Computacional e Algébrico na Formação Inicial de Professores de Matemática: Um Estudo de Caso com Scratch. Master's thesis, Universidade Estadual de Ponta Grossa.

Costa, L., Cavalcante, A., Araujo, A., Andrade, W., and Guerrero, D. (2017). Um Estudo Exploratório da Aplicação de Pensamento Computacional Baseado nas Perspectivas de Professores do Ensino Médio. Anais dos Workshops do Congresso Brasileiro de Informática na Educação, 6:992.

Echenique, M. T. and Fontes, R. D. (2020). A Formação de Professores para o Ensino de Computação na Educação Básica. Revista Eixo.

Eloy, A. A. d. S., Martins, A. R. Q., Pazinato, A. M., Lukjanenko, M. d. F. S. P., and Lopes, R. d. D. (2017). Programming Literacy: Computational Thinking in Brazilian Public Schools. Proceedings of the 2017 Conference on Interaction Design and Children, 10:439-444.

Farias, A., Andrade, W., and Alencar, R. (2015). Pensamento Computacional em Sala de Aula: Desafios, Possibilidades e a Formação Docente. Anais dos Workshops do Congresso Brasileiro de Informática na Educação, 4:1226.

França, R. and Tedesco, P. (2017). Pensamento Computacional sob a Perspectiva de Licenciandos em Computação. Anais do Workshop de Informática na Escola, 23:795804.

Gomes, C. A., Gomes, H., Rego, B., and Rito, P. N. (2020). Do Pensamento Computacional à Computação Criativa: Trajetórias na Formação Contínua de Professores. Mediações, 8(2):15-32.

Goulart, M., da Costa, P. K. A., and Pereira, A. (2019). A Integração das TDIC na Formação Inicial de Professores de Matemática no Brasil: Uma Análise a Partir dos Projetos Pedagógicos. Olhar de Professor, 21:351-367.

Júnior, P. A. P. and de Oliveira, S. (2019). Pensamento Computacional: Uma Proposta de Oficina para a Formação de Professores. Revista Novas Tecnologias na Educação, 17(01):62-71.

Kampff, A., Lopes, T., Alves, I., Souza, V., Rigo, S., and Marson, F. (2016). Pensamento Computacional no Ensino Superior: Relato de uma Oficina com Professores da Universidade do Vale do Rio dos Sinos. Anais dos Workshops do Congresso Brasileiro de Informática na Educação, 5:1316.

Kitchenham, B. (2004). Procedures for Performing Systematic Reviews. Technical report, Keele University - UK. 
Lira, A., Leitão, D., and Castro, J. (2019). Como o Processo de Produção de Mídias Pode Contribuir para a Formação Docente? Revista Novas Tecnologias na Educação, 17:425-434.

Macêdo, R. J. d. A., Cavallo, D. P., Gorender, S., and Araújo, B. M. d. (2020). Raciocínio Computacional na Formação Geral: Pavimentando o Caminho da Interdisciplinaridade. de Tughy, Rosângela Pereira; Gonçalves, Gustavo (Org.). Universidade popular e encontro de saberes. 1ed. Salvador: EDUFBA. Disponível em: https://repositorio.ufba.br/ri/handle/ri/32949. Acessado em 17/03/2021, 1:411-424.

Mandaji, M., Da Silva, R. K., Terçariol, A. A. D. L., and Mazurkievicz, G. L. (2018). O Programaê! e a Formação de Professores para a Integração do Pensamento Computacional ao Currículo. CIET:EnPED.

Marques, S. G., da Cruz, M. E. J. K., and Schulz, F. (2019). Formação Continuada de Licenciados em Computação para Trabalho com Computação na Escola. Anais dos Workshops do VIII Congresso Brasileiro de Informática na Educação, 8:31-40.

Martins, C. and Giraffa, L. (2020). Práticas Pedagógicas Remixadas: Relações entre Estratégias Pedagógicas da Cultura Digital e Formação Docente. Revista e-Curriculum, 18:739-760.

Matos, E., Coutinho, C., Santos, E., Dourado, H., Ribeiro, L., da Silva, R., de Jesus, S., Oliveira, T. K., and de Morais, P. (2019). Desafios da Tutoria em um Curso Online de Formação de Professores para Desenvolvimento do Raciocínio Computacional. Anais do IV Congresso sobre Tecnologias na Educação, 4:251-259.

Menolli, A., Bragagnolo, A., Gonçalves, R., and Neto, J. C. (2021). Factors Related to the Quality of Computer Science Teacher Education Courses: A Public Data Analysis. Revista Brasileira de Informática na Educação, 29(0):209-226.

Oliveira, C. and Pereira, R. (2019). Desenvolvimento do Pensamento Computacional no Ensino Superior em Ciência da Computação. Anais dos Workshops do Congresso Brasileiro de Informática na Educação, 8:1502.

Oliveira, E. and Bittencourt, R. (2019). Teaching Computational Thinking to K-12 Educators through Distance Learning. Anais do XXVII Workshop sobre Educação em Computação, 27:61-70.

Oliveira, E., Bittencourt, R., and Trindade, R. (2019). Designing and Evaluating a Computational Thinking Course for K-12 Brazilian Educators. Anais dos Workshops do Congresso Brasileiro de Informática na Educação, 8:1094.

Pasqual Júnior, P. A. (2018). Pensamento Computacional e Formação de Professores: Uma Análise a Partir da Plataforma Code.org. Master's thesis, Universidade de Caxias do Sul.

Paz, L. A. S. d. C. (2017). O Pensamento Computacional e a Formação Continuada de Professores: Uma Experiência com as TICs. Revista On-line de Política e Gestão Educacional, 22:1655-1667.

Pimentel, C. S., de Carvalho, N. A., and Barreiro, R. M. C. (2020). Aprendizado Ativo entre Bits e Átomos: Uma proposta para Formação de Professores no Contexto do Pensamento Computacional. VII Seminário Mídias e Educação, 1:31-42. 
Ramos, J. L. and Espadeiro, R. G. (2014). Os Futuros Professores e os Professores do Futuro: Os Desafios da Introdução ao Pensamento Computacional na Escola, no Currículo e na Aprendizagem. Revista Educação, Formação e Tecnologias, 7:4-25.

Reichert, J. T., Barone, D. A. C., and Kist, M. (2020). Computational Thinking in K-12: An analysis with Mathematics Teachers. Eurasia Journal of Mathematics, Science and Technology Education, 16(6).

SBC (2020). Itinerário Formativo da Computação. Sociedade Brasileira de Computação (SBC) - URL: https://www.sbc.org.br/documentos-da-sbc/send/203-educacaobasica/1216-itinerario-formativo-da-computacao - acessado em 17/03/2021.

Silva, J., Souza, S., Vanderlei, I., Andrade, M., and Alencar, A. (2019). Informática na Educação e Formação de Professores(as) da Rede Municipal de Garanhus - PE: Uma Experiência Extensionista. COINTER.

Silva, V., Silva, L., and França, R. (2017). Pensamento Computacional na Formação de Professores: Experiências e Desafios Encontrados no Ensino da Computação em Escolas Públicas. Anais do Workshop de Informática na Escola, 23:805-814.

Sokolonski, A. C., Sá, A., and Macedo, R. (2020). Robótica Educacional como Facilitadora do Aprendizado do Raciocínio Computacional: Revisão Sistemática da Literatura. Anais do XXXI Simpósio Brasileiro de Informática na Educação, 31:1503-1512.

Souza, A. d. G. (2019). Letramento Computacional no Ensino Superior: Reflexões e Aproximações do Saber/Fazer em Práticas de Pesquisa/Formação para os Anos Iniciais do Ensino Fundamental. PhD thesis, UNIVERSIDADE TIRADENTES.

Souza, I., Rodrigues, R., and Andrade, W. (2016). Introdução do Pensamento Computacional na Formação Docente para Ensino de Robótica Educacional. Anais dos Workshops do Congresso Brasileiro de Informática na Educação, 5:1265.

Valente, J. A. (2016). Integração do Pensamento Computacional no Currículo da Educação Básica: Diferentes Estratégias Usadas e Questões de Formação de Professores e Avaliação do Aluno. Revista E-curriculum, 14(3):864-897.

Vasconcelos, C. and Neto, A. J. M. (2018). A Formação de Professores de Computação para Adoção Apropriada de TDIC. CIET:EnPED.

von Wangenheim, A., von Wangenheim, C. G., Pacheco, F. S., Hauck, J. C. R., and Ferreira, M. N. F. (2017). Motivating Teachers to Teach Computing in Middle School: A Case Study of a Physical Computing Taster Workshop for K-12 Teachers. International Journal of Computer Science Education in Schools, 1(4):35-49.

Wing, J. (2006). Computational Thinking. Communications of the ACM, 49:33-35.

Zilio, C. and Nóbile, M. F. (2019). O Pensamento Computacional na Formação Continuada de Professores que Atuam no Laboratório de Informática. XII Encontro Nacional de Pesquisa em Educação em Ciências - UFRN. 